Bangladesh J. Plant Taxon. 15(1): 13-19, 2008 (June)

(C) 2008 Bangladesh Association of Plant Taxonomists

\title{
DIOECY AND MONOECY IN THE FLORA OF SRI LANKA AND THEIR EVOLUTIONARY CORRELATIONS TO ENDEMISM, GROWTH FORM, FRUIT TYPE, SEED NUMBER AND FLOWER SIZE
}

\author{
W.T.P.S.K. SENARATH ${ }^{1}$ \\ Department of Botany, University of Sri Jayewardenepura, Nugegoda, Sri Lanka
}

Keywords: Flora, Evolutionary correlations, Dioecy, Monoecy, Sri Lanka

\begin{abstract}
The frequency of dioecy and monoecy and the ecological correlates of each sexual system were examined for the flora of Sri Lanka, a tropical continental island. Of the 3,529 species in the total flora, $7.05 \%$ are dioecious, $10.25 \%$ are monoecious, $1.7 \%$ are polygamodioecious, $1.9 \%$ are polygamous and $79.1 \%$ are hermaphrodites. Study was carried out to test the null hypothesis that the proportionate distribution of dioecious and monoecious taxa in endemism, growth form, fruit type, seed number or flower size was not significantly different from that for the entire flora. Both monoecious and dioecious species in the Sri Lankan flora are significantly associated with the growth form, fruit type, seed number and flower size, whereas only dioecy is associated with endemism. Dioecious species are favored by trees and small flowers, whereas monoecious species are favored by herbs and minute flowers. Both dioecious and monoecious species are, however, associated with fleshy fruits suggesting that animal dispersal of fruits is favored in the Sri Lankan flora.
\end{abstract}

\section{Introduction}

Sri Lanka is a tropical continental island, which has a total area of about $65,610 \mathrm{sq}$ $\mathrm{km}$ of which total area under forest vegetation cover is 37.5\% (approx. 24,604 sq km). About $27 \%$ of land area (approx. 17,715 sq km) is covered by natural high forests (including mangroves). Natural vegetation type of the island is tropical humid forests. Out of the total 3,529 angiospermic species, $7.05 \%$ are dioecious, $10.25 \%$ are monoecious, $1.7 \%$ are polygamodioecious, $1.9 \%$ are polygamous and $79.1 \%$ are hermaphrodites (Abeywickrama 1956a). Sri Lanka separated from Indian landmass and existed as a separate island at the same time when the Himalayas arose as a land barrier in Miocene Epoch (10-27 million years ago). Isolation played an important role in evolving new forms or new species on this island. About $25 \%$ species of Sri Lankan flora is endemic, while remaining $75 \%$ non-endemic flora is found in South India, but nowhere else in the world (Abeywickrama 1956a).

There is a considerable disagreement about the selective forces that have led to the evolution of dioecy in plants. Selection to enforce out-crossing has historically being the primary explanation (Baker 1967, Charlesworth and Charlesworth 1978, Thomson and Barrett 1981). Tropical and island floras appear to have a large proportion of dioecious species than temperate and continental floras (Ashton 1969, Bawa 1980, Fanglian et al.

${ }^{1}$ E-mail: senerath@yahoo.co.uk 
1996). Dioecious flowers in temperate species are presumed to have evolved in response to selective pressure favoring wind pollination, yet wind pollination is probably either absent or uncommon in tropical species. Many of the species in tropical forests have unisexual flowers and animals as pollen vectors make it obvious that unisexual flowers in these species have not evolved in response to selective pressure for wind pollination as has been suggested for temperate plants (Bawa 1980). Selective forces favoring anemophily have been responsible for the evolution of dioecism (Bawa and Opler 1975). Despite the controversy that surrounds the ecology and evolution of dioecy (Givnish 1980), relatively few studies are available that examined either the frequency of dioecy in regional floras or the ecological correlates of dioecy (Flores and Schemske 1984). There are several evolutionary pathways to the dioecious condition. It can arise either from gynodioecious condition, monoecism, (Lewis 1942), heterostyly (Barker 1959) or from hermaphroditism (Barker 1959). The particular pathways through which a given taxon has evolved, dioecism can only be determined by comparative studies of taxa that contain related species with different breeding systems. It is not certain, on the other hand, if monoecism generally evolved from andromonoecism. There is a little discussion on different evolutionary pathways involved. Selective forces other than those associated with pollination may also play a role in the evolution of this sexual system (Bawa and Beach 1981).

Most of the dry zone species in low country and just under half of the species in the wet zone of Sri Lanka are named as peninsular species. The high proportion of peninsular species in the wet zone of Sri Lanka indicates that in an earlier period, free migration from one region to the other must have been possible although present conditions are very unfavorable for a direct migration from the wetter parts of Sri Lanka to similar regions of Peninsular India (Abeywickrama 1956a).

Floras of tropical islands, particularly which are rich in endemics and still retain ancient floras could provide valuable information on the evolutionary trends of their breeding system. Chief factors which determine the present distribution of species appear to be the degree of isolation and the tolerance to changes in the environment exhibited by

the individual species (Abeywickrama 1956a). Because of the isolation of Sri Lankan flora, particularly the concentration of endemics in the wet zone of the island, it can be used for the comparative studies of the evolution of dioecy and monoecy. Thus, distribution of monoecy and dioecy in relation to different factors, namely endemism, growth form, fruit type, seed number and flower size were studied for the entire Sri Lankan flora.

\section{Materials and Methods}

The relevant information on angiosperm species and their sexual systems were obtained from Trimen (1931), Abeywickrama (1959b) and Dassanayaka and Fosberg 
(1985). All the dioecious and monoecious species were classified under five categories and several groups under each category as tabulated in Table 1. For the entire flora, 61 polygamodioecious species were considered dioecious because the perfect flowers found in these species are often functionally unisexual (Flores and Schemske 1984). Similarly, 67 polygamous species were considered monoecious. Some species were not included under certain categories because of the lack of particular information concerning their reproductive characteristics.

Table 1. Classification of Sri Lankan species into several groups under five categories (distribution, growth form, fruit type, seed number and flower size).

\begin{tabular}{ll}
\hline Category & Group \\
\hline 1. Distribution & (i) Endemic, (ii) Non-endemic (including doubtful endemics) \\
2. Growth form & (i) Trees, (ii) Shrubs, (iii) Herbs (including epiphytes), (iv) Climbers \\
3. Fruit type & (i) Dry-dehiscent, (ii) Dry-indehiscent, (iii) Fleshy-dehiscent, (iv) Fleshy-indehiscent \\
4. Seed number & (i) One seeded-dry, (ii) Many seeded-dry, (iii) One seeded-fleshy, (iv) Many seeded-fleshy \\
5. Flower size & $\begin{array}{l}\text { (i) Minute (length }<0.62 \mathrm{~cm}),(\text { ii) Small }(0.62-3.75 \mathrm{~cm}),(\text { iii) Medium (3.76 - } 5.00 \mathrm{~cm}),(\text { iv) Large } \\
\end{array}$ \\
& $(>5.00 \mathrm{~cm})$
\end{tabular}

Distribution of dioecious and monoecious taxa in relation to five categories and corresponding groups (Table 1) was compared with those of the entire flora by Chisquare analysis (Equations 1-3, modified from William 1997). The null hypothesis for all comparisons was that the proportionate representation of dioecious or monoecious taxa in the various categories is not significantly different from the distribution within entire flora.

Proportion of a group in total flora $(\mathrm{A})=$ Total number of observed species in a group under a category / Total number of species in the flora

Equation 1

Expected species number of a group under dioecy (or monoecy) $=\mathrm{A} \times$ Total number of observed species in dioecious (or monoecious) flora

Equation 2

Chi square $\left(\lambda^{2}\right)=(\text { Observed proportion of a category - Expected proportion of such category })^{2} /$ Expected proportion of the category

Equation 3

\section{Results and Discussion}

The proportion of dioecious species among the endemics was higher than that of monoecious species in the total flora (Table 2). Therefore, there is a significant association between dioecy and endemism in the flora of Sri Lanka, but there is no significant association between monoecy and endemism. Temporal segregation of male and female phases can increase out-crossing probabilities in monoecious species. Although selection to increase out-crossing has been hypothesized as an important factor in the evolution of dioecy (Bawa 1980), many hermaphrodites or monoecious species are also highly out-crossed. If dioecy is favored on islands, following the hypothesis of Baker 
(1967), one would expect a higher incidence of dioecy among endemics as compared to widely distributed taxa (Bawa 1982). According to the results obtained in the present study, it could be suggested that dioecy is favored in the Sri Lankan flora.

Table 2. Correlation between endemism, growth form, fruit type, seed number and flower size to dioecy and monoecy of Sri Lankan flora.

\begin{tabular}{|c|c|c|c|c|c|c|c|}
\hline \multirow[t]{2}{*}{ Category/group } & \multirow{2}{*}{$\begin{array}{l}\text { Total no. of } \\
\text { spp. in the flora }\end{array}$} & \multicolumn{3}{|c|}{ Dioecy } & \multicolumn{3}{|c|}{ Monoecy } \\
\hline & & Observed & Expected & $\lambda^{2}$ & Observed & Expected & $\lambda^{2}$ \\
\hline \multicolumn{8}{|l|}{ Endemism } \\
\hline Endemic & 738 & $114(3.23 \%)$ & 52.07 & 93.1 & $86(2.43 \%)$ & 75.69 & 1.768 \\
\hline Non-endemic & 2791 & $135(3.82 \%)$ & 196.90 & $P<0.001$ & $276(7.8 \%)$ & 286.27 & $P=0.18$ \\
\hline \multicolumn{8}{|l|}{ Growth form } \\
\hline Tree & 774 & $157(4.44 \%)$ & 54.61 & & $131(3.71 \%)$ & 78.48 & \\
\hline Shrub & 664 & $27(0.02 \%)$ & 46.85 & 309.8 & $54(1.53 \%)$ & 67.32 & 53.72 \\
\hline Herb & 1805 & $23(0.65 \%)$ & 127.34 & $P<0.001$ & $134(3.79 \%)$ & 183.03 & $P<0.001$ \\
\hline Climber & 285 & $42(1.19 \%)$ & 20.10 & & $39(1.1 \%)$ & 28.89 & \\
\hline \multicolumn{8}{|l|}{ Fruit type } \\
\hline \multicolumn{8}{|l|}{ Dry } \\
\hline Dehiscent & 1143 & $57(1.62 \%)$ & 99.86 & & $124(3.53 \%)$ & 141.7 & \\
\hline Indehiscent & 1112 & $84(2.39 \%)$ & 76.95 & 41.07 & $48(1.36 \%)$ & 109.19 & 107.3 \\
\hline Fleshy & & & & $P<0.001$ & & & $P<0.003$ \\
\hline Dehiscent & 71 & $4(0.11 \%)$ & 4.91 & & $17(0.48 \%)$ & 6.97 & \\
\hline Indehiscent & 886 & $98(0.79 \%)$ & 61.31 & & $156(4.44 \%)$ & 87 & \\
\hline \multicolumn{8}{|l|}{ Seed number } \\
\hline \multicolumn{8}{|l|}{ One seeded } \\
\hline Fleshy & 194 & $6(0.17 \%)$ & 13.40 & & $55(1.56 \%)$ & 19.03 & \\
\hline Dry & 971 & $55(1.56 \%)$ & 67.09 & 1119.6 & $36(1.02 \%)$ & 95.25 & 2056.49 \\
\hline Many seeded & & & & $P<0.001$ & & & $P<0.001$ \\
\hline Fleshy & 771 & $96(2.70 \%)$ & 53.28 & & $118(3.35 \%)$ & 75.63 & \\
\hline Dry & 1580 & $86(2.44 \%)$ & 5.94 & & $136(3.86)$ & 8.44 & \\
\hline \multicolumn{8}{|l|}{ Flower size } \\
\hline Minute & 1140 & $84(2.42 \%)$ & 80.59 & & $235(6.79 \%)$ & 118.20 & \\
\hline Small & 1707 & $143(4.12 \%)$ & 120.68 & 15.26 & $117(3.37 \%)$ & 117.00 & 176.15 \\
\hline Medium & 254 & $5(0.14 \%)$ & 17.95 & $P>0.001$ & $3(0.08 \%)$ & 26.33 & $P<0.001$ \\
\hline Large & 262 & $13(0.38 \%)$ & 18.52 & & $4(0.11 \%)$ & 27.17 & \\
\hline
\end{tabular}

The results obtained from the present study indicated that there is a highly significant association of dioecy and monoecy with growth form. Trees proportionally included more dioecious species, whereas herbs included more monoecious species (Table 2). Moreover, the expected proportions of shrubs and herbs were higher than that of observed proportions, but the expected proportions of climbers were lower than that of observed proportions in both dioecy and monoecy. The ratio of dioecious: monoecious species was 1.19 for trees, 0.5 for shrubs, 0.17 for herbs and 1.07 for climbers. These data 
indicate that the relative frequency of dioecy and monoecy declines sharply with decreasing size of the growth form. According to the evolutionary history, the first angiosperms are believed to have been woody, with adaptable herbaceous forms arising soon thereafter. Trees have the highest incidence of dioecy and herbs the lowest (Bawa 1980), which is comparable with the results of the present study. Out-breeding mechanisms are a feature of perennial plants and their high incidence in trees is wellknown (Bawa 1982). This high incidence is also related to the evolutionary advantages of out-crossing in terms of genetic flexibility (Bawa and Beach 1981).

For the entire flora of Sri Lanka, observed number of dioecious and monoecious species with fleshy fruits is more than twice than that of expected (Table 2). There is a significant association between dioecy and monoecy to fruit type (dry- or fleshydehiscent or indehiscent). The expected and observed proportions of fleshy-indehiscent fruits are more favored than fleshy-dehiscent fruits. Moreover, there is a significant association between seed number and dioecy or monoecy. The expected proportion of dry, many-seeded fruits is lower than observed proportion in both dioecious and monoecious species, whereas expected proportion of dry one-seeded is higher than that of observed proportion only in dioecy. If the dispersal efficiency of one- and many-seeded dry fruits is same, according to the null hypothesis distribution of these characters among entire flora should be in same proportions. For the entire flora, observed proportion of fleshy many-seeded fruits is comparatively higher than that of fleshy one-seeded fruits, explaining fleshy many-seeded fruits are favored in the process of evolution. In dioecious tropical trees and shrubs the seeds in the vast majority of species are dispersed by birds. It is noteworthy that dispersal by birds is more common in tropical than temperate plant communities (Carlquist 1974). Long distance dispersal of plants by birds is an important source of colonization of species on oceanic islands. Thus the highest incidence of dioecy in tropical flora could be partly due to correlation between dioecy and dispersal by birds (Givnish 1980).

There is a significant association found between monoecy and size of the flower, whereas the association between dioecy and flower size is not significant (Table 2). To investigate this association, when flowers were placed in four classes it was observed that among dioecious species, small flowers are favored, but minute flowers are favored among monoecious species.

The evolution of angiosperms is closely linked with insects and the flowers of the angiosperms arose in all probability from an etomophilous (insect-pollinated) strobilus. Wind-pollinated species may have then arose from insect-pollinated species. Minute flowers are more adapted for insect as well as wind pollination, but small flowers are adapted only to insect pollination (Baker 1967). As in both monoecious and dioecious species observed proportions of minute and small flowers are higher than medium or large flowers it could be concluded that wind- or insect-pollinated flowers are more 
favored than beetle- or bird-pollinated flowers among the Sri Lankan flora or the evolution of dioecy and monoecy among Sri Lankan flora is more effected by minute or small flowers.

The Sri Lankan flora also showed similarities with the flora of other tropical islands. For example, the percentage of dioecy and monoecy in flora of Barro Colorado Island (9.0\% and $15.7 \%$ in Croat 1979$)$ and Puerto Rico $(6.1 \%$ and $10.5 \%$ in Flores and Schemske 1984) were comparable to that of Sri Lankan estimates (7.05\% and 10.25\%). In terms of growth form, the flora of Barro Colorado Island (Croat 1979) demonstrated increase in the frequency of dioecy with an increase in the size of plants as did Sri Lankan flora.

\section{Acknowledgements}

Author would like to thank Prof. I.A.U.N. Gunathillake of the University of Peradeniya, Sri Lanka and Dr. Magdon Jayasuriya, Former Curator of the Royal Botanic Gardens, Sri Lanka for their guidance throughout this study.

\section{References}

Abeywickrama, B.A. 1956a. The origin and affinities of the flora of Ceylon. Proc. $11^{\text {th }}$ Ann. Ses. Cey. Ass. Adv. Sci., pp. 99-121.

Abeywickrama, B.A. 1956b. A Check List of the Angiosperms in Sri Lanka. NARESA publication, Sri Lanka, pp. 1-125.

Asthon, P.S. 1969. Speciation among tropical forest trees: some deductions in the light of recent evidence. Biol. J. Linn. Soc. 1: 155-196.

Barker, H.G. 1959. Reproductive methods as factors in speciation of flowering plants. Cold Spring Harbor Symposium on Quantiative Biology 24: 177-191.

Baker, H.G. 1967. Support for Baker's law - as a rule. Evolution 21: 853-856.

Bawa, K.S. 1980. Evolution of dioecy in flowering plants. Ann. Rev. Ecol. System. 11: 15-40.

Bawa, K.S. 1982. Outcrossing and the incidence of dioecism in island floras. Am. Nat. 119: 866-871.

Bawa, K.S. and Beach, J.H. 1981. Evolution of sexual systems in flowering plants. Ann. Mis. Bot. Gar. 68: 254-274.

Bawa, K.S. and Opler, P.A. 1975. Dioecism in tropical forest trees. Evolution 29: 167-179.

Carlquist, S. 1974. Island Biology. Columbia University Press, New York, pp. 286-295.

Charlesworth, B. and Charlesworth, D. 1978. A model for the evolution of dioecy and gynodioecy. Am. Nat. 112: $975-997$.

Croat, T.B. 1979. The sexuality of the Barro Colorado Island flora (Panama). Phyto. 42: 319-348.

Dassanayaka, M.D. and Fosberg, F.R. 1985. Revised Handbook to the Flora of Sri Lanka. Vols I-V. Amarind Publishers Corporation (pvt) Ltd., New Delhi.

Fangliang, H., Legendre, P. and Frankie, J.L. 1996. Spatial pattern of diversity in a tropical rain forest in Malaysia. J. Biogeography 23: 57-74.

Flores, S. and Schemske, D.W. 1984. Dioecy and monoecy in the flora of Puerto Rico and the Virgin Islands: ecological correlates. Biotropica 16: 132-139. 
DIOECY AND MONOECY IN THE FLORA OF SRI LANKA

Givnish, T.J. 1980. Ecological constraints in the evolution of breeding systems in seed plants: dioecy and dispersal in gymnosperms. Evolution 34: 959-972.

Lewis, D. 1942. The evolution of sex in flowering plants. Biological Rev. Cam. Phil. Soc. 17: 46-67.

Thomson, J.D. and Barrett, S.C.H. 1981. Selection for outcrossing, sexual selection, and the evolution of dioecy in plants. Am. Nat. 118: 443-449.

Trimen, H. 1931. A Handbook to the Flora of Ceylon. Vols I-VI. Dalau C. Ltd., London.

William, P.G. 1997. Statistics for Bioscience. Prentice Hall, Englewood Cliff, NJ, pp. 76-85.

(Manuscript received on 12 September 2007; revised on 19 December 2007) 